\title{
On the Statistical Origin of Topological Symmetries
}

\author{
K. Aghababaei Samani ${ }^{a}$ and A. Mostafazadeh ${ }^{b}$ \\ ${ }^{a}$ Institute for Advanced Studies in Basic Sciences, \\ 45195-159 Gava Zang, Zanjan, IRAN* \\ ${ }^{b}$ Department of Mathematics, Koç University, \\ Rumelifeneri Yolu, 80910 Sariyer, Istanbul, TURKEY ${ }^{\dagger}$
}

\begin{abstract}
We investigate a quantum system possessing a parasupersymmetry of order 2 , an orthosupersymmetry of order $p$, a fractional supersymmetry of order $p+1$, and topological symmetries of type $(1, p)$ and $(1,1, \cdots, 1)$. We obtain the corresponding symmetry generators, explore their relationship, and show that they may be expressed in terms of the creation and annihilation operators for an ordinary boson and orthofermions of order $p$. We give a realization of parafermions of order 2 using orthofermions of arbitrary order $p$, discuss a $p=2$ parasupersymmetry between $p=2$ parafermions and parabosons of arbitrary order, and show that every orthosupersymmetric system possesses topological symmetries. We also reveal a correspondence between the orthosupersymmetry of order $p$ and the fractional supersymmetry of order $p+1$.
\end{abstract}

\section{Introduction}

During the past two decades supersymmetry has been widely used in a number of problems in quantum mechanics [2, 3, 活. This has been one of the main reasons for the development and the study of various generalizations of supersymmetric quantum mechanics (SQM). Among the best known examples of these generalizations are the parasupersymmetric

\footnotetext{
*E-mail address: samani@iasbs.ac.ir

${ }^{\dagger}$ E-mail address: amostafazadeh@ku.edu.tr
} 
quantum mechanics (PSQM) [5, 6, 7, 8, 9], the fractional supersymmetric quantum Mechanics (FSQM) [10, 11, 12], and the orthosupersymmetric quantum mechanics (OSQM) [13. Recently, we have introduced a class of generalizations of SQM that unlike the above mentioned generalizations

share the topological properties of supersymmetry in the sense that these symmetries involve a set of integer-valued topological invariants. We have termed these symmetries Topological Symmetries. In Refs. [14, 15], we develop the concept of a topological symmetry (TS), obtain the underlying operator algebra, and show that the algebras of SQM, PSQM of order 2, and FSQM of arbitrary order are special cases of the algebras of different types of TSs. An important difference between our approach to TSs and the standard approach to SQM and PSQM is that we do not define TS as a generalization of the symmetries of an oscillator consisting of two degrees of freedom with specific statistics. The purpose of the present article is to seek for such a statistical interpretation of TS.

It is well-known that algebras of SQM [16, 2], PSQM [5, 8, 9], and OSQM [13] have been originally obtained as symmetries of an oscillator consisting of a bosonic degree of freedom and a fermionic, a parafermionic, or an orthofermionic degree of freedom, respectively. Other realizations of SQM in a pure parafermionic system, a pure parabosonic system, and a parafermi-parabose oscillator have also been discussed in the literature [17, 18, 19]. Moreover, a $k$-fermionic realization of PSQM is offered in Ref. [20].

The algebra of FSQM has been originally obtained as a simple but rather ad hoc generalization of the algebra of SQM that was mainly influenced by the ideas of $q$-deformed bosons [10, 11, 12. A somewhat concrete justification for the basic algebraic relations used in FSQM is given in Ref. [21]. The idea of finding a realization of FSQM using an oscillator consisting of a boson and another particle has been pursued in Refs. [12, 22].

In this paper we consider a quantum mechanical system whose Hamiltonian has a parasupersymmetry of order 2 , a fractional supersymmetry of order $p+1$, an orthosupersymmetry of order $p$, and TSs of type $(1, p)$ and $(1,1, \cdots, 1)$. We show that the generators of these symmetries have a statistical interpretation in terms of bosons and orthofermions.

The organization of the paper is as follows. In Section 2, we review topological sym- 
metries. In Section 3, we survey the algebras of PSQM, OSQM, and FSQM. In Section 4, we use a concrete system to investigate the statistical origin of these symmetries and their relationship. In Section 5, we show that every OSQM system possesses TSs of type $(1, p)$ and $(1,1, \cdots, 1)$, and construct the generators of TSs in terms of the generators of OSQM. In Section 6, we give a summary of our results and present our concluding remarks.

\section{Topological Symmetries}

A topological symmetry is a generalization of supersymmetry in the sense that it allows for the introduction of certain topological invariants. These are the analogues of the Witten index of SQM [1]. Here we give the definition and the operator algebra of the topological symmetries. For more details we refer the reader to Ref. 15.

A quantum system is said to possess a $\mathbb{Z}_{n}$-graded (uniform) topological symmetry (UTS) of type $\left(m_{1}, m_{2}, \cdots, m_{n}\right)$ iff the following conditions are satisfied.

1. The quantum system is $\mathbb{Z}_{n}$-graded. This means that the Hilbert space $\mathcal{H}$ of the quantum system is the direct sum of $n$ of its (nontrivial) subspaces $\mathcal{H}_{\ell}$, and its Hamiltonian has a complete set of eigenvectors with definite color or grading. (A state is said to have a definite color $c_{\ell}$ iff it belongs to $\left.\mathcal{H}_{\ell}\right)$;

2. The energy spectrum is nonnegative;

3. Every positive energy eigenvalue $E$ is $m$-fold degenerate, and the corresponding eigenspaces are spanned by $m_{1}$ vectors of color $c_{1}, m_{2}$ vectors of color $c_{2}, \cdots$, and $m_{n}$ vectors of color $c_{n}$.

For a system with these properties we can introduce a set of integer-valued topological invariant, namely

$$
\Delta_{i j}:=m_{i} n_{j}^{(0)}-m_{j} n_{i}^{(0)}
$$

where $i, j=1, \cdots, n$ and $n_{\ell}^{(0)}$ denotes the number of zero-energy states of color $c_{\ell}$, 15. Note that the TS of type $(1,1)$ coincides with supersymmetry and $\Delta_{11}$ yields the Witten index. 
As shown in Ref. [15, one can obtain the underlying operator algebra of TSs. For a

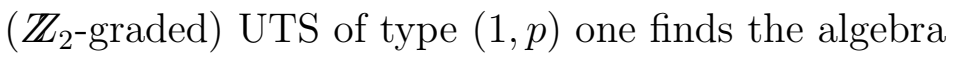

$$
\begin{aligned}
& {[H, \mathcal{Q}]=0,} \\
& \left\{\mathcal{Q}^{2}, \mathcal{Q}^{\dagger}\right\}+\mathcal{Q} \mathcal{Q}^{\dagger} \mathcal{Q}=2 H \mathcal{Q}, \\
& \mathcal{Q}^{3}=0 \\
& {[H, \tau]=\{\tau, \mathcal{Q}\}=0} \\
& \tau^{2}=1, \quad \tau^{\dagger}=\tau,
\end{aligned}
$$

where $H$ is the Hamiltonian of the system, $\mathcal{Q}$ is the generator of UTS, and $\tau$ is the grading operator. We can also express these algebraic relation in terms of Hermitian generators:

$$
Q_{1}:=\frac{1}{\sqrt{2}}\left(\mathcal{Q}+\mathcal{Q}^{\dagger}\right) \quad \text { and } \quad Q_{2}:=\frac{-i}{\sqrt{2}}\left(\mathcal{Q}-\mathcal{Q}^{\dagger}\right)
$$

This yields

$$
Q_{1}^{3}=Q_{1} H \quad \text { and } \quad Q_{2}^{3}=Q_{2} H
$$

The algebra of $\left(\mathbb{Z}_{n}\right.$-graded) UTS of type $(\underbrace{1,1, \cdots, 1}_{n \text { times }})$ is given by

$$
\begin{aligned}
& \mathcal{Q}^{n}=K \\
& Q_{1}^{n}+M_{n-2} Q_{1}^{n-2}+\cdots=\left(\frac{1}{\sqrt{2}}\right)^{n}(2 K), \\
& Q_{2}^{n}+M_{n-2} Q_{2}^{n-2}+\cdots=\left(\frac{1}{\sqrt{2}}\right)^{n}\left(i^{n}+(-i)^{n}\right) K \\
& {[\tau, \mathcal{Q}]_{q}=0 .}
\end{aligned}
$$

where $M_{i}$ s and $K$ are Hermitian operators commuting with all other operators, and $\tau$ is the grading operator satisfying

$$
\begin{aligned}
\tau^{n} & =1, \\
\tau^{\dagger} & =\tau^{-1}, \\
{[H, \tau] } & =0 .
\end{aligned}
$$

Here $[., .]_{q}$ stands for $q$-commutation defined by $\left[O_{1}, O_{2}\right]_{q}:=O_{1} O_{2}-q O_{2} O_{1}$, and $q:=e^{2 \pi i / n}$. One should note that this is not the most general form of the algebra of UTS of type $(1,1, \cdots, 1)$, but this form suffices for the purposes of this article. 


\section{Para, Ortho and Fractional Supersymmetry}

In this section we survey the algebras of quantum systems with parasupersymmetry of order 2 , orthosupersymmetry of order $p$, and fractional supersymmetry of order $n$.

\subsection{Algebra of the PSQM of order 2}

A $p=2$ PSQM system is defined by the following algebra [5].

$$
\begin{aligned}
& {[H, \mathcal{P}]=0} \\
& \left\{\mathcal{P}^{2}, \mathcal{P}^{\dagger}\right\}+\mathcal{P} \mathcal{P}^{\dagger} \mathcal{P}=4 H \mathcal{P} \\
& \mathcal{P}^{3}=0
\end{aligned}
$$

Here $\mathcal{P}$ is the generator of the parasupersymmetry. Comparing Eqs. (16) - (18) with Eqs. (2) - (4), we see that the algebra of $p=2$ PSQM and UTS of type $(1, p)$ are identical. This does not, however, imply that any $p=2$ parasupersymmetric system possesses a UTS of type $(1, p)$. The key observation is that the defining properties of UTS of type $(1, p)$ lead to the algebra $(\sqrt[16]{16})$ - $(\sqrt{18})$, but this algebra does not imply the defining degeneracy structure of the UTS of type $(1, p)$.'

\subsection{Algebra of the OSQM of order $p$}

The algebra of an OSQM of order $p$ is given by

$$
\begin{aligned}
& {\left[H, \mathcal{Q}_{\alpha}\right]=0,} \\
& \mathcal{Q}_{\alpha} \mathcal{Q}_{\beta}^{\dagger}+\delta_{\alpha \beta} \sum_{\gamma=1}^{p} \mathcal{Q}_{\gamma}^{\dagger} \mathcal{Q}_{\gamma}=2 \delta_{\alpha \beta} H \\
& \mathcal{Q}_{\alpha} \mathcal{Q}_{\beta}=0
\end{aligned}
$$

where $\mathcal{Q}_{\alpha}$, with $\alpha=1,2, \cdots, p$, are the generators of the orthosupersymmetry [13].

\subsection{Algebra of the FSQM of order $n$}

FSQM of order $n$ is defined by the relation

$$
\mathcal{F}^{n}=H
$$

\footnotetext{
${ }^{1}$ The set of $p=2$ parasupersymmetric systems which do satisfy the defining conditions of UTS of type $(1, p)$ form a proper subset of all the $p=2$ parasupersymmetric systems, 23].
} 
where $\mathcal{F}$ is the generator of fractional supersymmetry [10, 11]. Note that $\mathcal{F}$ need not be Hermitian. Furthermore, as suggested by the algebra (9) - (12) of the $\mathbb{Z}_{n}$-graded UTS of type $(1,1, \cdots, 1)$, any system having a $\mathbb{Z}_{n}$-graded UTS of type $(1,1, \cdots, 1)$ also has a fractional supersymmetry of order $n$ [15].

\section{Statistical Origin of TS in a Simple Toy Model}

Consider a quantum system with the Hamiltonian

$$
H=a^{\dagger} a+\sum_{\gamma=1}^{p} c_{\gamma}^{\dagger} c_{\gamma},
$$

where $a$ is the annihilation operator for a bosonic degree of freedom satisfying

$$
\left[a, a^{\dagger}\right]=1
$$

and $c_{\gamma}$, with $\gamma=1, \cdots, p$, are annihilation operators of orthofermions of order $p$ satisfying 13

$$
\begin{aligned}
& c_{\alpha} c_{\beta}^{\dagger}+\delta_{\alpha \beta} \sum_{\gamma=1}^{p} c_{\gamma}^{\dagger} c_{\gamma}=\delta_{\alpha \beta}, \\
& c_{\alpha} c_{\beta}=0 .
\end{aligned}
$$

A simple representation of $c_{\alpha}$ is given by the $(p+1) \times(p+1)$ matrices

$$
\left[c_{\alpha}\right]_{i j}=\delta_{i, 1} \delta_{j, \alpha+1}, \quad i, j=1, \cdots, p+1 .
$$

In this representation, the Hamiltonian of the system reads

$$
H=\operatorname{diag}(a^{\dagger} a, \underbrace{a a^{\dagger}, \cdots, a a^{\dagger}}_{p \text { times }})
$$

where 'diag $(\cdots)$ ' stands for 'diagonal matrix with diagonal elements ...'.

It is easy to check that the Hamiltonian (23) and the operator

$$
\mathcal{Q}_{\alpha}=\sqrt{2} a^{\dagger} c_{\alpha}, \quad \alpha=1, \cdots, p
$$

satisfy the algebra (19) - (21) of OSQM.2

\footnotetext{
${ }^{2}$ Note that we postulate relative bosonic statistics between bosons and orthofermions, i.e., $\left[a, c_{\alpha}\right]=0$ for all $\alpha$.
} 
Next, consider the operator

$$
\mathcal{P}=\frac{2}{\sqrt{p}}\left(a \sum_{j=1}^{r} c_{\gamma_{j}}^{\dagger}+a^{\dagger} \sum_{j=r+1}^{p} c_{\gamma_{j}}\right),
$$

where $\left(\gamma_{1}, \cdots \gamma_{p}\right)$ is an arbitrary permutation of $(1, \cdots, p)$ and $r$ is an integer between 1 and $p-1$. It turns out that the Hamiltonian (23) and the operator $\mathcal{P}$ fulfil the algebra (16) $-(18)$ of $p=2$ PSQM. Clearly, $\mathcal{P}$ is determined by the choice of the permutation $\left(\gamma_{1}, \cdots \gamma_{p}\right)$. Therefore this system has many parasupersymmetries of order 2 .

In the next section, we shall prove that in general every orthosupersymmetric quantum mechanical system possesses a $p=2$ parasupersymmetry. Parasupersymmetry was originally introduced as a symmetry of a bosonic-parafermionic oscillator [0]. However, there are also other statistical interpretations for PSQM. An example is a realization of the parasupersymmetry algebra using $k$-fermions [20]. Another example is a paraboseparafermi parasupersymmetry of the Hamiltonian:

$$
H=\frac{1}{2}\left\{\tilde{a}^{\dagger}, \tilde{a}\right\}+\frac{1}{2}\left[b^{\dagger}, b\right]
$$

where $\tilde{a}$ is the annihilation operator of a paraboson of arbitrary order $p=1,2, \cdots$ satisfying

$$
\left[\tilde{a}^{2}, \tilde{a}^{\dagger}\right]=2 \tilde{a}
$$

and $b$ is the annihilation operator of a parafermion of order 2 satisfying

$$
\begin{aligned}
& b^{3}=0, \\
& b^{\dagger} b^{2}+b^{2} b^{\dagger}=b b^{\dagger} b=2 b .
\end{aligned}
$$

The Hamiltonian (31) and the operator

$$
\mathcal{P}=\tilde{a} b^{\dagger}
$$

fulfil the algebra of PSQM of order 2, namely Eqs. (16) - (18). [?

It is well-known that one can construct parafermionic and parabosonic operators using the Green's ansatz [24]. The above relationship between $p=2$ parasupersymmetry and

\footnotetext{
${ }^{3}$ Here we postulate relative bosonic statistics between parafermions and parabosons, i.e., $[\bar{a}, b]=$ $\left[\bar{a}, b^{\dagger}\right]=0$.
} 
orthosupersymmetry suggests the following construction of $p=2$ parafermions in terms of orthofermions:

$$
b=\sum_{j=1}^{r} \alpha_{j} c_{\gamma_{j}}^{\dagger}+\sum_{j=r+1}^{p} \alpha_{j} c_{\gamma_{j}},
$$

where $1 \leq r<p$ and $\alpha_{i}$ satisfy

$$
\sum_{j=1}^{r}\left|\alpha_{j}\right|^{2}=\sum_{j=r+1}^{p}\left|\alpha_{j}\right|^{2}=2 .
$$

For example, we may set

$$
\alpha_{i}=\left\{\begin{array}{ccc}
\sqrt{\frac{2}{r}} & \text { for } & 1 \leq i \leq r \\
\sqrt{\frac{2}{p-r}} & \text { for } & r+1 \leq i \leq p .
\end{array}\right.
$$

It is not difficult to show that $b$ as given by Eq. (36) satisfies the algebra of $p=2$ parafermions, namely Eqs. (33) and (34).

Furthermore, we can also check that the Hamiltonian (23) with the operator $\mathcal{Q}=$ $\mathcal{P} / \sqrt{2}$ satisfy both the algebra (2) - (5) and the defining conditions for the UTS of type $(1, p)$. The corresponding grading operator is given by

$$
\tau=(-1)^{N}, \quad \text { where } \quad N:=\sum_{\alpha=1}^{p} N_{\alpha},
$$

and $N_{\alpha}$ are orthofermionic number operators:

$$
N_{\alpha}:=c_{\alpha}^{\dagger} c_{\alpha} .
$$

Next, we note that the Hamiltonian (31) has a fractional supersymmetry of order $p+1$ generated by

$$
\mathcal{F}=a c_{1}^{\dagger}+c_{2}^{\dagger} c_{1}+\cdots+c_{p}^{\dagger} c_{p-1}+a^{\dagger} c_{p} .
$$

Finally, this Hamiltonian has, in addition, a $\mathbb{Z}_{p+1}$-graded UTS of type $(1,1, \cdots, 1)$ with the same generator as $\mathcal{F}$ and the grading operator

$$
\tau=q^{\mathcal{N}}, \quad \text { where } \quad \mathcal{N}:=\sum_{\alpha=1}^{p} \alpha N_{\alpha},
$$

the operator $K$ of Eq. (9) is identified with the Hamiltonian $H$, the operators $M_{i}$ of Eqs. (10) and (11) are given in terms of $H$ according to

$$
M_{n-2 k}=(-1)^{k}\left[\frac{1}{2^{k}}\left(\begin{array}{c}
n-k-1 \\
k
\end{array}\right)+\frac{1}{2^{k-1}}\left(\begin{array}{c}
n-k-1 \\
k-1
\end{array}\right) H\right],
$$

and $\left(\begin{array}{c}a \\ b\end{array}\right):=\frac{a !}{b !(a-b) !}$. 


\section{Relation between General OSQM and TSs}

In this section we show that orthosupersymmetric systems always possess TSs of type $(1, p)$ and $(1,1, \cdots, 1)$.

Consider an arbitrary orthosupersymmetric Hamiltonian $H$ satisfying Eqs. (19) - (21) and let

$$
\mathcal{Q}:=\frac{1}{\sqrt{p}}\left(\sum_{j=1}^{r} \mathcal{Q}_{\gamma_{j}}^{\dagger}+\sum_{j=r+1}^{p} \mathcal{Q}_{\gamma_{j}}\right),
$$

where $\left(\gamma_{1}, \cdots, \gamma_{p}\right)$ is a permutation of $(1, \cdots, p)$. Then, it can be easily checked that $\mathcal{Q}$ is a generator for a TS of type $(1, p)$. The $\mathbb{Z}_{2}$-grading of the Hilbert space is established via the grading operator

$$
\tau=(-1)^{\left(\frac{\mathcal{Q}_{1} \mathcal{Q}_{1}^{\dagger}}{2 H}\right)}=1-\frac{\mathcal{Q}_{1} \mathcal{Q}_{1}^{\dagger}}{H}
$$

Furthermore, the Hamiltonian (20) can be expressed in terms of the generators $\mathcal{Q}_{\gamma}$ according to

$$
H=\frac{1}{2}\left(\mathcal{Q}_{1} \mathcal{Q}_{1}^{\dagger}+\sum_{\gamma=1}^{p} \mathcal{Q}_{\gamma}^{\dagger} \mathcal{Q}_{\gamma}\right)
$$

where we have used the fact that $\mathcal{Q}_{1} \mathcal{Q}_{1}^{\dagger}=\cdots=\mathcal{Q}_{p} \mathcal{Q}_{p}^{\dagger}$.

Next, we use the generators of the orthosupersymmetry $\mathcal{Q}_{\gamma}$ to construct a generator for the TS of type $(1,1, \cdots, 1)$ for this system, namely

$$
\mathcal{Q}=\mathcal{Q}_{1}^{\dagger}+\mathcal{Q}_{2}^{\dagger} \mathcal{Q}_{1}+\cdots+\mathcal{Q}_{p}^{\dagger} \mathcal{Q}_{p-1}+\mathcal{Q}_{p}
$$

This operator together with the Hamiltonian (46) and the grading operator

$$
\tau=(q)^{\sum_{\alpha=1}^{p} \frac{\alpha \mathcal{Q}_{\alpha}^{\dagger} \mathcal{Q}_{\alpha}}{2 H}}=1+\frac{1}{2 H} \sum_{\alpha=1}^{p} \mathcal{Q}_{\alpha}^{\dagger} \mathcal{Q}_{\alpha}\left(q^{\alpha}-1\right)
$$

satisfy the algebra (9) - (15) of the $\mathbb{Z}_{p+1}$-graded TS of type $(1,1, \cdots, 1)$. The operators $K$ and $M_{n-2 k}$ appearing in this algebra are given by

$$
\begin{aligned}
& K=(2 H)^{p} \\
& M_{n-2 k}=(-2)^{k} H^{2 k-1}\left[\left(\begin{array}{c}
n-k-1 \\
k
\end{array}\right) H+\left(\begin{array}{c}
n-k-1 \\
k-1
\end{array}\right)\right] .
\end{aligned}
$$

In particular, $K$ may be viewed as the Hamiltonian of a fractional supersymmetric system. Therefore, to every orthosupersymmetric system of order $p$, there corresponds a fractional supersymmetric system of order $p+1$.

\footnotetext{
${ }^{4}$ This is actually to be expected for the system has the required degeneracy structure 13 .
} 


\section{Concluding Remarks}

In this article, we have explored the statistical origins of certain topological symmetries. We have obtained an interpretation of topological symmetries of type $(1, p)$ and $(1,1, \cdots, 1)$ in terms of certain symmetries between bosons and orthofermions. We have given a realization of $p=2$ parafermions in terms of orthofermions of arbitrary order, and revealed a novel relationship between fractional supersymmetry and orthosupersymmetry. We have also shown that every orthosupersymmetric system has topological symmetries

of type $(1, p)$ and $(1,1, \cdots, 1)$. Our results have potential application in the construction of quantum systems possessing topological symmetries and having a topologically nontrivial configuration space. The study of these systems is of particular importance for a better understanding of the mathematical meaning of the topological invariants $\Delta_{i j}$. 


\section{References}

[1] E. Witten, Nucl. Phys. B 202, 253 (1982).

[2] L. E. Gendenshtein and I. V. Krive, Sov. Phys. Usp. 28, 645-666 (1985)

[3] F. Cooper, A. Khare, and U. Sukhatme, Phys. Rep. 251, 267-385 (1995).

[4] G. Junker, Supersymmetric Methods in Quantum and Statistical Physics (SpringerVerlag, Berlin, 1996).

[5] V. A. Rubakov and V. P. Spiridonov, Mod. Phys. Lett. A 3, 1337 (1988).

[6] J. Beckers and N. Deberg, Nucl. Phys. B 340, 767 (1990).

[7] S. Durand, M. Mayrand and V. Spridonov, Mod. Phys. Lett. A 6, 3163 (1991)

[8] M. Tomiya, J. Phys. A: Math. Gen. 25, 4699 (1992);

A. Khare, J. Phys. A: Math. Gen. 25, L749 (1992).

[9] A. Khare, J. Math. Phys. 34, 1277 (1993)

[10] C. Ahn, D. Bernard, and A. Leclair, Nucl. Phys. B 346, 409 (1990);

L. Baulieu and E. G. Floratos, Phys. Lett. B 258, 171 (1991);

R. Kerner, J. Math. Phys. 33, 403 (1992);

S. Durand, Mod. Phys. Lett. A 8, 1795 (1993);

S. Durand, Mod. Phys. Lett. A 8, 2323 (1993);

A. T. Filippov, A. P. Isaev, and R. D. Kurdikov, Mod. Phys. Lett. A 7, 2129 (1993);

N. Mohammedi, Mod. Phys. Lett. A 10, 1287 (1995);

N. Fleury and M. Rausch de Traubenberg, Mod. Phys. Lett. A 11, 2899 (1996).

[11] S. Durand, Phys. Lett. B 312, 115 (1993).

[12] J. A. de Azćarraga and A. Macfarlane, J. Math. Phys. 37, 1115 (1996);

R. S. Dunne, A. Macfarlane, J. A. de Azćarraga, and J. C. Pérez Bueno, Int. J. Mod. Phys. Lett. A 12, 3275 (1997). 
[13] A. Khare, A. K. Mishra and G. Rajasekaran, Int. J. Mod. Phys. A 8,1245 (1993).

[14] A. Mostafazadeh and K. Aghababaei Samani, Mod. Phys. Lett. A 15, 175 (2000).

[15] K. Aghababaei Samani and A. Mostafazadeh , Nucl. Phys. B 595,467 (2001).

[16] B. DeWitt, Supermanifolds, 2nd Ed. (Cambridge Uni. Press, Cambridge, 1992).

[17] S. Klishevish and M. Plyushchay, Mod. Phys. Lett. A 39, 2739 (1999).

[18] M. Plyushchay, Int. J. Mod. Phys. Lett. A 15, 3679 (2000).

[19] A. Mostafazadeh, Int. J. Mod. Phys. Lett. A 17, 2957 (1996), See also J. Beckers and N. Deberg, J. Math. Phys. 31, 1513 (1991).

[20] M. Daoud and M. R. Kibler 'A Fractional Supersymmetric Oscillator and its Coherent States,' math-ph/9912024.

[21] A. T. Filippov and A. B.Kurdikov, 'Paragrassmann Algebras with Many Variables,' hep-th/9312081.

[22] M. Daoud and M. R. Kibler, 'On Fractional Supersymmetric Quantum Mechanics: The Fractional Supersymmetric Oscillator,' math-ph/0101009.

[23] A. Mostafazadeh, Int. J. Mod. Phys. Lett. A 12, 2725 (1997).

[24] H. S. Green, Phys. Rev. 90, 270 (1953). 